\title{
Design of a Novel Miniaturized Frequency Selective Surface Based on 2.5-Dimensional Jerusalem Cross for 5G Applications
}

\author{
Peng Zhao, ${ }^{1,2}$ Yihang Zhang $\mathbb{D}^{1}{ }^{1}$ Rongrong Sun, ${ }^{1}$ Wen-Sheng Zhao, ${ }^{1}$ \\ Yue $\mathrm{Hu}^{1}{ }^{1}$ and Gaofeng Wang ${ }^{1}{ }^{1}$ \\ ${ }^{1}$ Key Lab of RF Circuits and Systems of Ministry of Education, School of Electronics and Information, Hangzhou Dianzi University, \\ Hangzhou 310018, China \\ ${ }^{2}$ State Key Laboratory of Millimeter Waves, Southeast University, Nanjing 211189, China
}

Correspondence should be addressed to Gaofeng Wang; gaofeng@hdu.edu.cn

Received 21 December 2017; Accepted 8 March 2018; Published 19 April 2018

Academic Editor: Ting-Yen Shih

Copyright (C) 2018 Peng Zhao et al. This is an open access article distributed under the Creative Commons Attribution License, which permits unrestricted use, distribution, and reproduction in any medium, provided the original work is properly cited.

\begin{abstract}
A compact frequency selective surface (FSS) for 5G applications has been designed based on 2.5-dimensional Jerusalem cross. The proposed element consists of two main parts: the successive segments of the metal traces placed alternately on the two surfaces of the substrate and the vertical vias connecting traces. Compared with previous published two-dimensional miniaturized elements, the transmission curves indicate a significant size reduction (1/26 wavelengths at the resonant frequency) and exhibit good angular and polarization stabilities. Furthermore, a general equivalent circuit model is established to provide direct physical insight into the operating principle of this FSS. A prototype of the proposed FSS has been fabricated and measured, and the results validate this design.
\end{abstract}

\section{Introduction}

In recent years, frequency selective surfaces (FSSs) have drew extensive attentions because of their wide applications in the communication equipment $[1,2]$. FSSs are always designed to reflect, transmit, or absorb electromagnetic wave, and they are applied in the design of antenna radomes, reflector of low-profile antenna, electromagnetic absorbers, and so on [3-7]. The typical FSS structures consist of two-dimensional (2D) periodically arranged resonate units [8-10]. In practical design, the number of FSS units is restricted to the requirement of size. For $5 \mathrm{G}$ communications, the antennas are very small and the radomes covering the antennas should be small as well [11]. However, when the infinite period of the FSS is truncated, it is bound to have a significant impact on the performance of the FSS. In order to maintain original performances and compromise this constraint, compact FSS elements are required.

At present, several methods are proposed to realize the miniaturization of FSS. By adding some lumped reactive components to their design, the size of FSS element has been reduced because of the increasement of the inductance and capacitance of equivalent circuit [12]. In [13], the miniaturization of element is realized by reducing the thickness of dielectric substrate, so that the capacitance between various metallic layers has been increased. Moreover, a loop-wire structure has been introduced in [14], which consists of a metallic patch and wire-grid on the opposite layer of substrate to enhance the inductance and capacitance. The FSS element consists of four symmetrical spiral patterns of metallic meander lines which has been designed to increase the length of resonant structures in a given periodicity [15]. In [16], the spiral slot element with a compact arrangement has been proposed, which effectively increases the corresponding equivalent inductance. And the corresponding equivalent capacitance can also be increased by adjusting the distance between every two slots. More recently, square loop with vertical vias is applied to design FSS element [17]. In this design, the capacitance is increased by the capacitive coupling of adjacent via wall, and the inductance is increased by using the knitted structure alternately across different layers of substrate. The element size of this design is around 1/16 wavelengths. For 5G mobile communications, further size reduction should be investigated. 


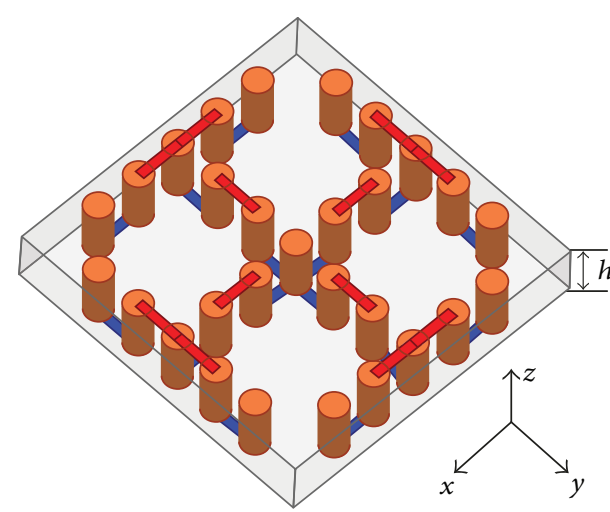

(a)

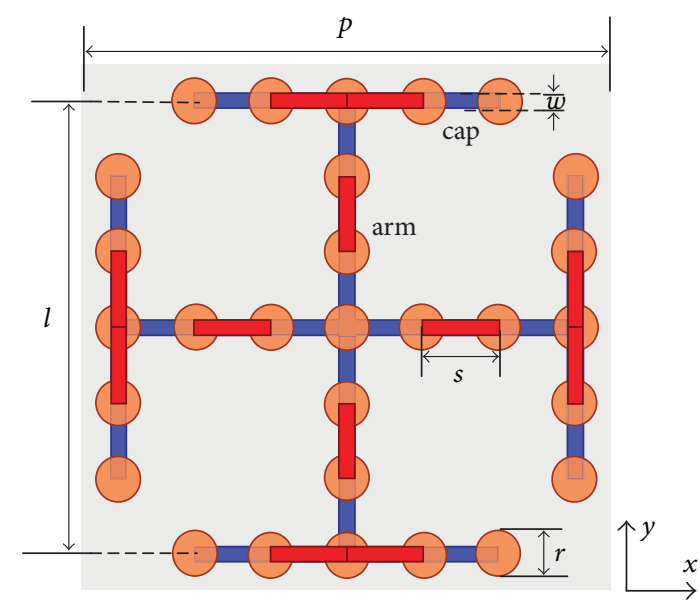

(b)

FIGURE 1: Geometry of the 2.5D straight-line type FSS element: (a) 3D perspective view and (b) top view.

In this paper, novel miniaturized FSSs based on $2.5 \mathrm{D}$ Jerusalem cross element have been designed, and the working frequency is around $3.3 \mathrm{GHz}$ which is the potential $5 \mathrm{G}$ communication frequency. The FSS element uses 41 vias to provide additional inductance and capacitance due to extensive path and via wall coupling between adjacent elements. Comparing with [17], this design has more significant effect of size reduction which achieves a FSS element around 1/26 wavelengths at the resonant frequency. Moreover, this FSS provides good resonant stability for various polarizations and incident angles. The equivalent circuit model of the FSS has also been proposed for the analysis of its performance. Finally, to validate the results of proposed FSS, a prototype has been fabricated and measured. The proposed 2.5D FSS can be easily fabricated using low-cost PCB processing. The results show a good consistency between the full-wave simulations and measurements.

\section{Element Design and Performance Analysis}

The FSS element can be treated as a resonance circuit when it is illuminated by the incident waves. The resonant frequency is determined by the formula $f=1 /(2 \pi \sqrt{L C})$, where $L$ and $C$ represent equivalent inductance and capacitance of the element, respectively. Therefore, the method of miniaturization technique is to increase the value of inductance and capacitance. Based on this theory, the proposed structure of the $2.5 \mathrm{D}$ miniaturized element has been designed.

In Figure 1, the metallic segments of the straight-line type FSS element are alternately placed on the top and bottom surface of the substrate and then connected through the metallic vias. The element is printed on a dielectric substrate FR-4 $\left(\varepsilon_{r}=4.4\right.$; loss tangent $\left.\delta=0.025\right)$, and the periodicity of unit cell is $p$. The length of the planar element is $l$ and the width of its conductor is $w$. Each arm and cap are divided into five and seven segments, respectively. And then, the successive segments are printed on the top and bottom side of substrate connected through vias alternatively. The radius of all vias is $r$ while the center to center spacing between adjacent
TABLE 1: Dimension of the FSS unit cell.

\begin{tabular}{lc}
\hline Parameter & Value $(\mathrm{mm})$ \\
\hline$p$ & 3.5 \\
$l$ & 3 \\
$w$ & 0.1 \\
$s$ & 0.5 \\
$h$ & 1 \\
$r$ & 0.15 \\
\hline
\end{tabular}

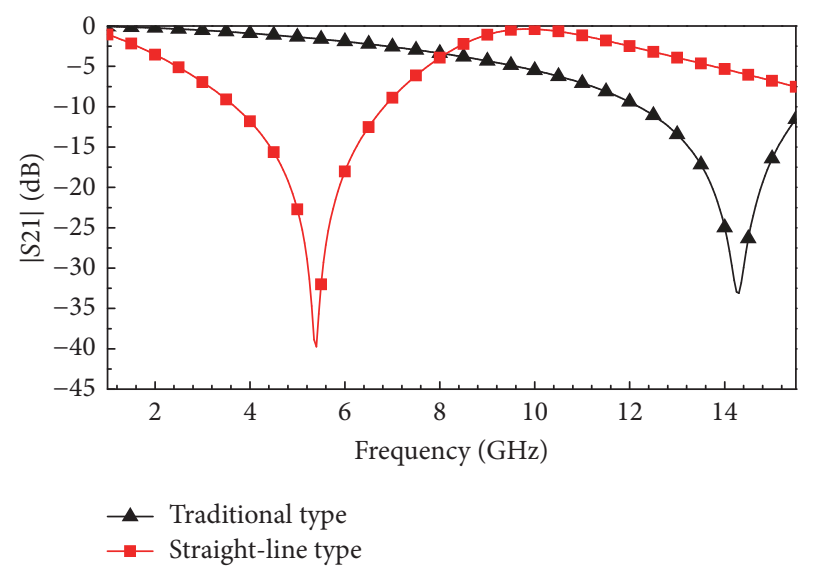

FIGURE 2: Transmission coefficients of the proposed FSS with straight-line arm with $h=1 \mathrm{~mm}$ for normally incident TE-polarized plane wave.

vias is $s$. The dimensions of the designed FSS are listed in Table 1.

It can be observed from Figure 2 that the straight-line type element resonates at $5.5 \mathrm{GHz}$ in contrast to the traditional Jerusalem cross element with the same design parameters that has a resonant frequency of $14.5 \mathrm{GHz}$. Thus, the size of the proposed element has been reduced from 1/6 to 1/16 wavelengths. 
TABLE 2: Comparison of element sizes.

\begin{tabular}{|c|c|c|c|c|c|}
\hline Element & Period $(\mathrm{mm})$ & Thickness (mm) & Dielectric constant & Resonant frequency & Size \\
\hline Ref. [12] & 8.85 & 0.8 & 4.4 & $0.935 \mathrm{GHz}$ & $0.028 \lambda_{0}$ \\
\hline This FSS & 3.5 & 0.8 & 4.4 & $3.88 \mathrm{GHz}$ & $0.045 \lambda_{0}$ \\
\hline Ref. [13] & 7.5 & 0.9 & 2.65 & $1.39 \mathrm{GHz}$ & $0.035 \lambda_{0}$ \\
\hline This FSS & 3.5 & 0.9 & 2.65 & $4.48 \mathrm{GHz}$ & $0.052 \lambda_{0}$ \\
\hline Ref. [15] & 4.4 & 1 & 4.4 & $3.3 \mathrm{GHz}$ & $0.048 \lambda_{0}$ \\
\hline This FSS & 3.5 & 1 & 4.4 & $3.3 \mathrm{GHz}$ & $0.038 \lambda_{0}$ \\
\hline Ref. [16] & 4.8 & 1.6 & 4.4 & $3.82 \mathrm{GHz}$ & $0.063 \lambda_{0}$ \\
\hline Ref. [17] & 10 & 1.6 & 4.4 & $1.89 \mathrm{GHz}$ & $0.0625 \lambda_{0}$ \\
\hline This FSS & 3.5 & 1.6 & 4.4 & $2.29 \mathrm{GHz}$ & $0.026 \lambda_{0}$ \\
\hline
\end{tabular}

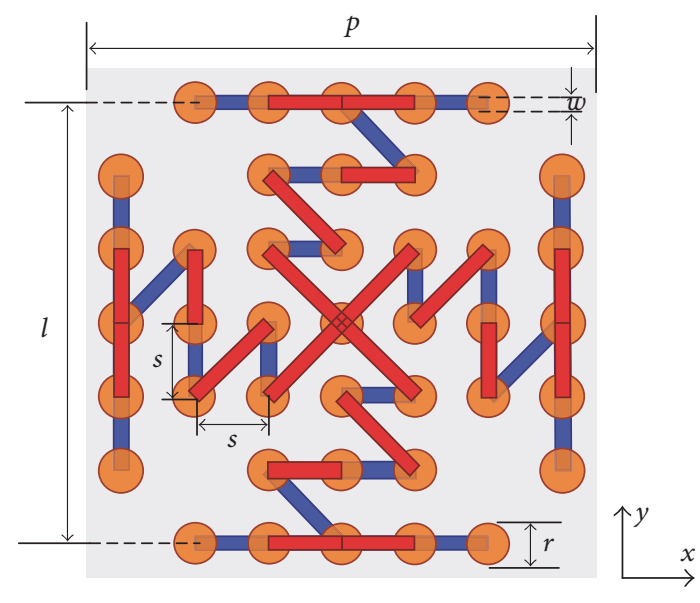

FIGURE 3: Geometry of the 2.5D meander-line type FSS element (top view).

To further miniaturize the size of element, a 2.5D Jerusalem cross with meander-line arm is proposed, as shown in Figure 3. Comparing with the previous structure, the proposed element with inserted additional vias and metal traces has a longer electric length. Therefore, the value of equivalent inductance can be increased and the resonant frequency can be decreased as well. Figure 4 shows that the transmission coefficients of the proposed FSS. The performance is simulated using finite element method incorporating with periodic boundary condition. The meander-line type element resonates at the frequency of $3.3 \mathrm{GHz}$ with the same substrate thickness. Correspondingly, the period of the meandering line element is reduced to $1 / 26$ wavelengths. Hence, the size of element has been reduced considerably. Furthermore, the transmission response with various substrate thicknesses has been studied. With the reduction of substrate thickness, the resonant frequency decreases due to the reduction of the inductance and capacitance of the metallic vias.

In Figure 5, the angular and polarization stabilities of proposed FSS element have been investigated. From this figure, it is observed that the maximum deviation is about $0.3 \%$ between the incidence angles $0^{\circ}$ and $60^{\circ}$ in both the TE and TM cases, which indicates extremely stable resonance at various polarizations and incidence angles.

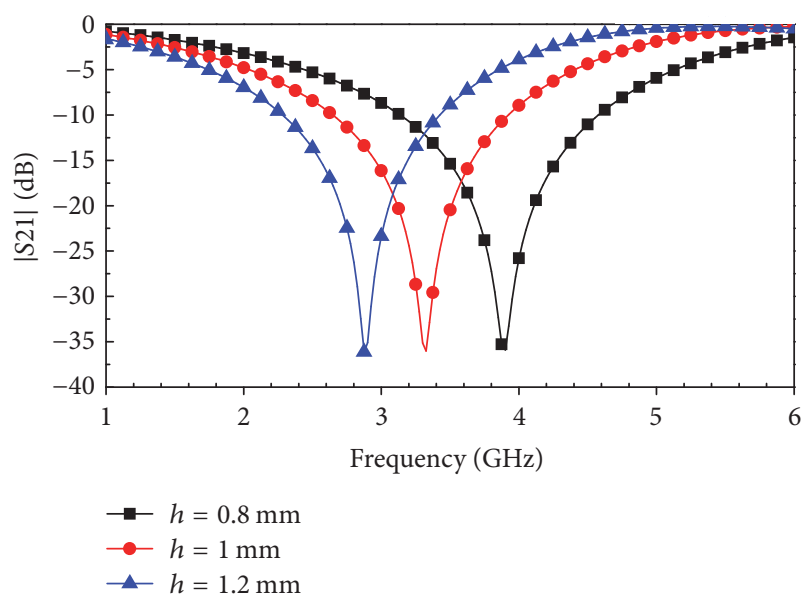

FIgURE 4: Transmission coefficients of the meander-line type FSS with various substrate thicknesses for normally incident TEpolarized plane wave.

A size comparison between the proposed and previous reported FSSs is provided in Table 2. For a fair comparison, the same substrate as that in the papers is adopted. It can be seen that the size of proposed FSS is very small. Although the size of FSS in [12] is smaller, it uses bulky lumped reactive components for the miniaturization. Similarly, the FSSs in [13] are two-dielectric layer structure. Compared with the designs on single dielectric layer [15-17], the proposed FSS has significant size reduction.

\section{Equivalent Circuit Models Validation and Results Analysis}

To provide direct physical insight into the operating principle of the 2.5D Jerusalem cross FSS, an equivalent circuit model is proposed in this section. The traditional Jerusalem cross is described by series $L C$ circuit model. For the 2.5D Jerusalem cross, metal vias in the substrate can be treated as an additional inductor to link the top and bottom strips together, and the adjacent vias in two neighbor units can be treated as an additional capacitor, respectively.

As shown in Figure 6(a), $L_{1}$ and $L_{v}$ represent the equivalent inductance, respectively, generated by the serial 


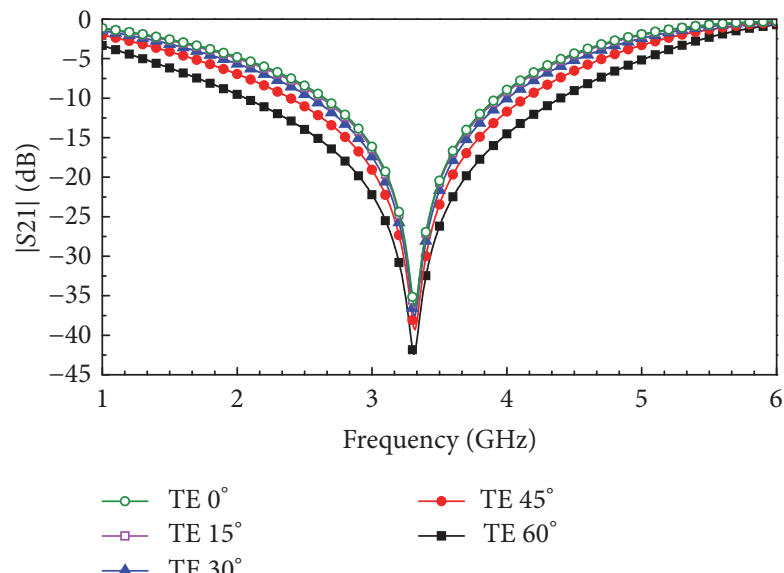

(a)

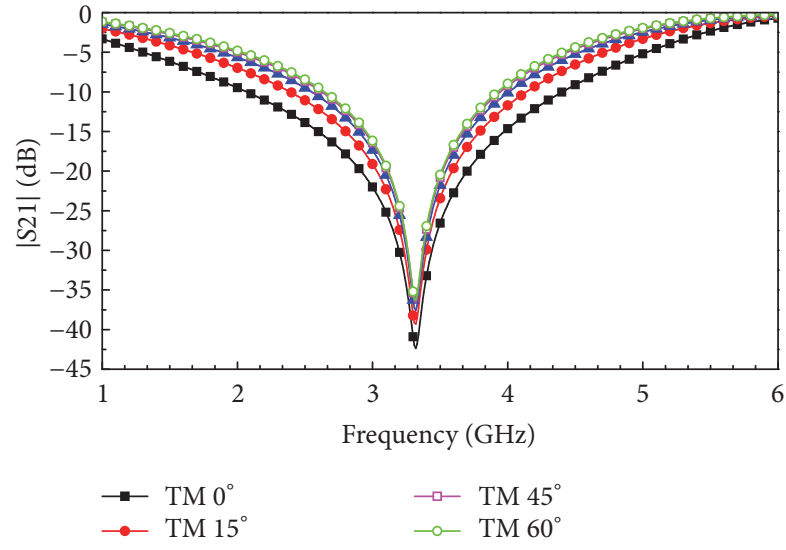

(b)

FIGURE 5: Simulated transmission coefficients of proposed FSS ( $h=1 \mathrm{~mm}$ ): (a) illuminated by normally incident TE-polarized wave of different incident angles; (b) illuminated by normally incident TM-polarized wave of different incident angles.
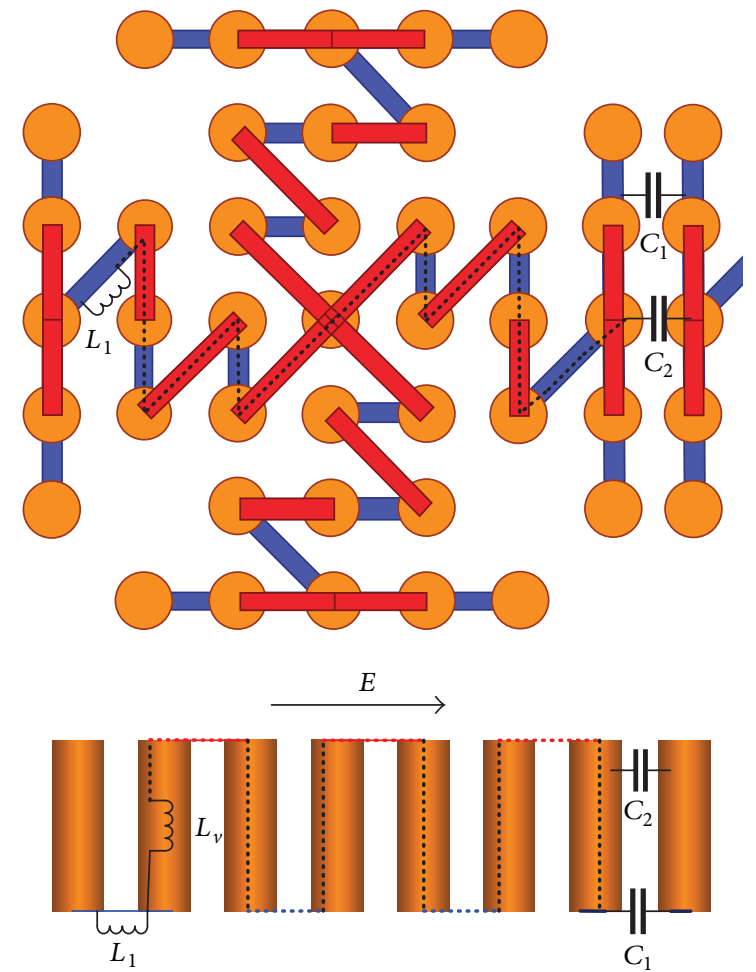

Metal tracks at top layer

Metal tracks at bottom layer

Metal vias

(a)

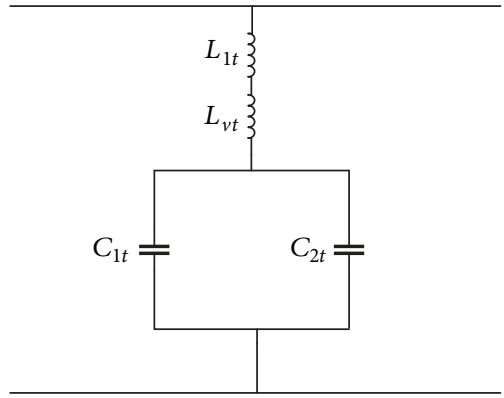

(b)

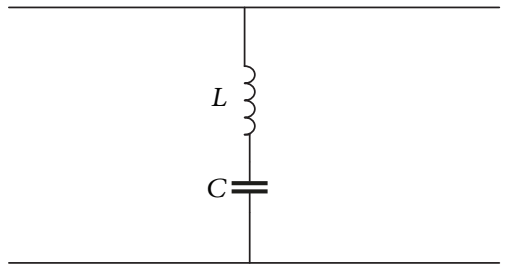

(c)

FIGURE 6: The equivalent circuit of proposed FSS element. 


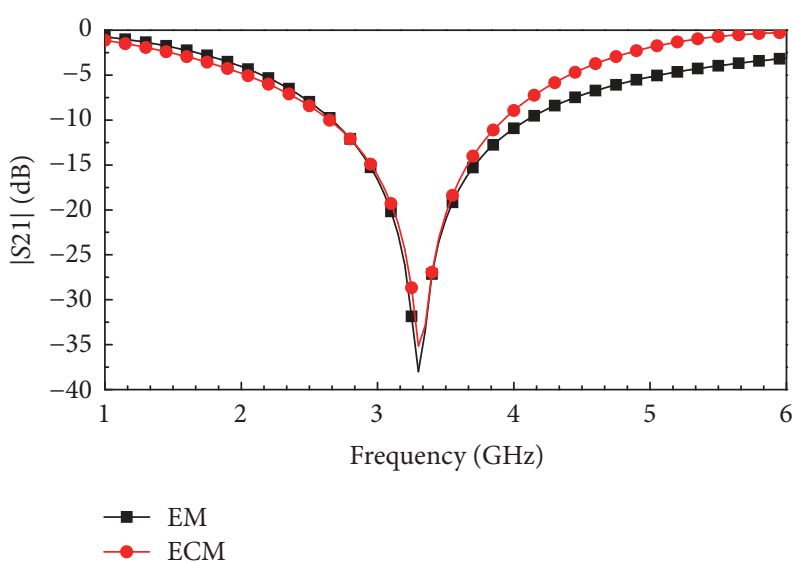

Figure 7: Transmission coefficients of 2.5D FSS comparison of equivalent circuit model and electromagnetic simulation.

metal strips and vertical vias, while $C_{1}$ and $C_{2}$ represent the equivalent capacitance, respectively, generated by the adjacent metal strips and vertical vias in two neighbor units. The equivalent circuit of the FSS element is illustrated in Figure 6(b) in which the total contribution of serial metal strips and vias for the inductance is represented by $L_{1 t}$ and $L_{v t}$. Similarly, the total contribution of adjacent metal strips and vertical vias for the capacitance is represented by $C_{1 t}$ and $C_{2 t}$. By combining the inductances $L_{1 t}$ and $L_{v t}$ into $L$ and combining capacitances $C_{1 t}$ and $C_{2 t}$ into $C$, the FSS element can be described by a simpler equivalent circuit, as shown in Figure 6(c).

The equivalent inductance and capacitance of this circuit model depend on the given physical parameters. The equivalent inductance can be increased by adding substrate thickness or decreasing the spacing between adjacent vias because of the increasing electrical length. Similarly, the equivalent capacitance can be increased by narrowing the gap between neighboring element. Therefore, the resonant frequency can be tuned by adjusting the relevant parameters of the FSS element.

In order to validate the methodology of the proposed equivalent circuit, its performance is assessed against the results obtained from full-wave electromagnetic simulations. As shown in Figure 7, the transmission curves of the equivalent circuit model and electromagnetic have been illustrated. It can be observed that the frequency responding of equivalent circuit model is accurate with the electromagnetic simulations, though its bandwidth evaluation becomes slightly narrower compared with electromagnetic simulation.

\section{Fabrication and Measurement}

In order to validate the designed structures in the above sections, the proposed FSS is fabricated using the normal PCB technique and tested in a free-space measurement environment. The design parameters of these prototype are the same as those given in Section 2. The fabricated FSS sample is shown in Figure 8. The FSS fabricated on FR-4

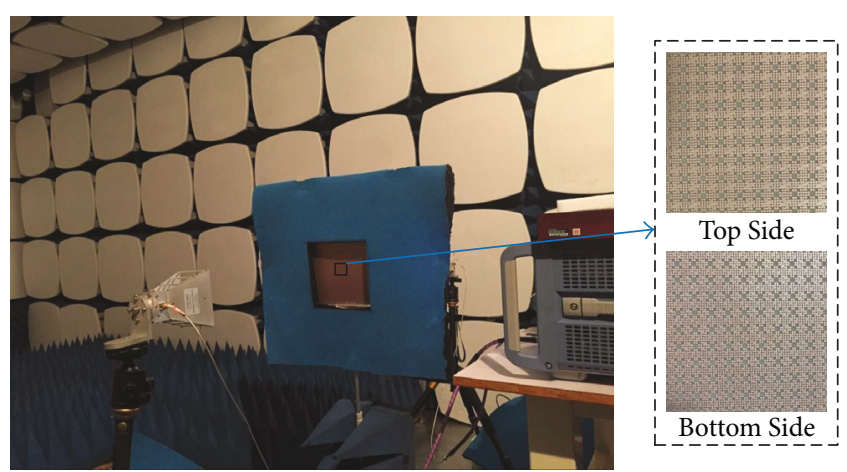

FIGURE 8: Measurement setup for the proposed FSS.

board and the total size is $350 \times 350 \mathrm{~mm}^{2}$ and consists of 100 $\times 100$ elements. The FSS is measured in a microwave anechoic chamber with a vector network analyzer and two standard horn antennas are placed on the two sides of the FSS as the transmitting and receiving antennas.

Figure 9 shows the transmission coefficients at various incident angles, with both TE and TM polarizations. It can be observed that the measured results are evidently consistent with the proposed models. In addition, the measurement results confirmed that the proposed FSS exhibited a stable resonant frequency. The discrepancies between the measurements and the simulations are mainly caused by the fabrication tolerance of the fine metal lines and vias and measurement uncertainties. Overall, the experimental results can demonstrate the performance of the proposed miniaturized FSS.

\section{Conclusion}

A novel miniaturized FSS based on 2.5D Jerusalem cross has been designed for the application to $5 \mathrm{G}$ antenna radomes. Benefitting from the additional inductance and capacitance due to the $2.5 \mathrm{D}$ structure, the unit length of this FSS is only $1 / 26$ wavelengths at the resonant frequency of $3.3 \mathrm{GHz}$. Furthermore, the resonant frequency of the proposed FSS exhibited a good stability at various polarizations and incident angles. The deviation of the resonant frequency is less than $0.3 \%$ even if the incident angle is $60^{\circ}$ in both TE and TM cases. Moreover, an equivalent circuit model has been proposed to provide direct physical insight. The experiments' results have been given and these results validate the proposed design.

\section{Conflicts of Interest}

The authors declare that there are no conflicts of interest regarding the publication of this paper.

\section{Acknowledgments}

This work was supported in part by the National Key R\&D Program of China under Grant 2017YFB0203500, in part by the National Natural Science Foundation of China under 


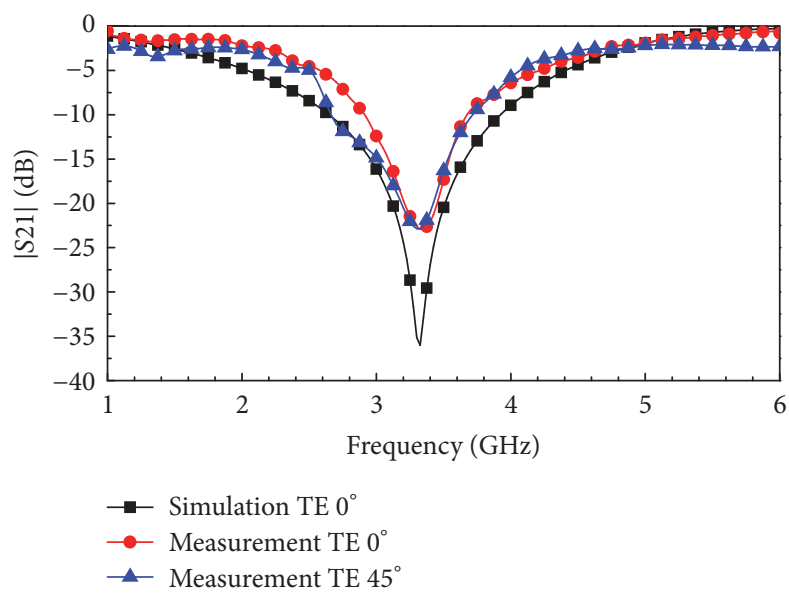

(a)

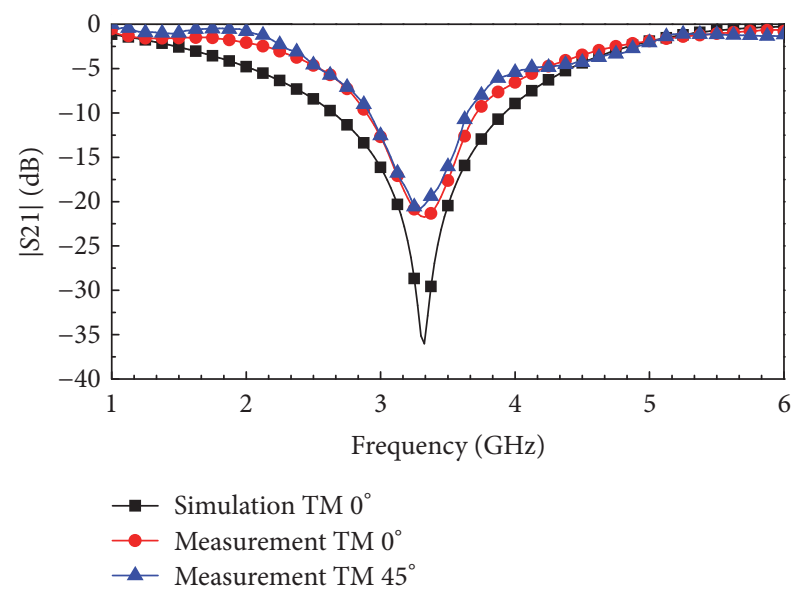

(b)

FIGURE 9: Transmission coefficients of the proposed FSS obtained from measurements and EM simulations with various incident angles: (a) for TE polarization with a substrate thickness of $1 \mathrm{~mm}$; (b) for TM polarization with a substrate thickness of $1 \mathrm{~mm}$.

Grants 61601163, 61331007, 61504033, and 61411136003, and in part by the State Key Laboratory of Millimeter Waves under Grant K201710.

\section{References}

[1] B. A. Munk, Frequency selective surface: Theory and design, Wiley, New York, NY, USA, 2000.

[2] R. Mittra, C. H. Chan, and T. Cwik, "Techniques for Analyzing Frequency Selective Surfaces-a Review," Proceedings of the IEEE, vol. 76, no. 12, pp. 1593-1615, 1988.

[3] C.-C. Chen, "Transmission of Microwave Through Perforated Flat Plates of Finite Thickness," IEEE Transactions on Microwave Theory and Techniques, vol. 21, no. 1, pp. 1-6, 1973.

[4] F. Birbir, J. Shaker, and Y. M. M. Antar, "Chebishev bandpass spatial filter composed of strip gratings," IEEE Transactions on Antennas and Propagation, vol. 56, no. 12, pp. 3707-3713, 2008.

[5] J. H. Kim, H. J. Chun, I. P. Hong, Y. J. Kim, and Y. B. Park, "Analysis of FSS radomes based on physical optics method and ray tracing technique," IEEE Antennas and Wireless Propagation Letters, vol. 13, pp. 868-871, 2014.

[6] N. M. Mohamed-Hicho, E. Antonino-Daviu, M. CabedoFabres, and M. Ferrando-Bataller, "A novel low-profile highgain UHF antenna using high-impedance surfaces," IEEE Antennas and Wireless Propagation Letters, vol. 14, pp. 1014-1017, 2015.

[7] J. Lee, M. Yoo, and S. Lim, "A study of ultra-thin single layer frequency selective surface microwave absorbers with three different bandwidths using double resonance," Institute of Electrical and Electronics Engineers. Transactions on Antennas and Propagation, vol. 63, no. 1, pp. 221-230, 2015.

[8] T. K. Wu, Frequency Selective Surfaces and Grid Array, Wiley, Hoboken, NJ, USA, 1995.

[9] J. C. Vardaxoglou, Frequency Selective Surfaces Analysis and Design, Wiley, Hoboken, NJ, USA, 1997.

[10] C.-N. Chiu, Y.-C. Chang, H.-C. Hsieh, and C. H. Chen, "Suppression of spurious emissions from a spiral inductor through the use of a frequency-selective surface," IEEE Transactions on Electromagnetic Compatibility, vol. 52, no. 1, pp. 56-63, 2010.
[11] W. Yadum and N. Nakajima, "The correlation of diversity/MIMO antenna for portable terminals," Wireless Communications and Mobile Computing, vol. 7, no. 8, pp. 995-1002, 2007.

[12] H. L. Liu, K. L. Ford, and R. J. Langley, "Design methodology for a miniaturized frequency selective surface using lumped reactive components," IEEE Transactions on Antennas and Propagation, vol. 57, no. 9, pp. 2732-2738, 2009.

[13] B.-Q. Lin, S.-H. Zhou, X.-Y. Da, Y.-W. Fang, Y.-J. Li, and W. Li, "Compact miniaturised-element frequency selective surface," IEEE Electronics Letters, vol. 51, no. 12, pp. 883-884, 2015.

[14] F. Bayatpur and K. Sarabandi, "Multipole spatial filters using metamaterial-based miniaturized-element frequency-selective surfaces," IEEE Transactions on Microwave Theory and Techniques, vol. 56, no. 12, pp. 2742-2747, 2008.

[15] M. Yan, S. Qu, J. Wang et al., "A novel miniaturized frequency selective surface with stable resonance," IEEE Antennas and Wireless Propagation Letters, vol. 13, pp. 639-641, 2014.

[16] G. Yang, T. Zhang, W. Li, and Q. Wu, "A novel stable miniaturized frequency selective surface," IEEE Antennas and Wireless Propagation Letters, vol. 9, pp. 1018-1021, 2010.

[17] T. Hussain, Q. Cao, J. K. Kayani, and I. Majid, "Miniaturization of Frequency Selective Surfaces Using 2.5-D Knitted Structures: Design and Synthesis," IEEE Transactions on Antennas and Propagation, vol. 65, no. 5, pp. 2405-2412, 2017. 


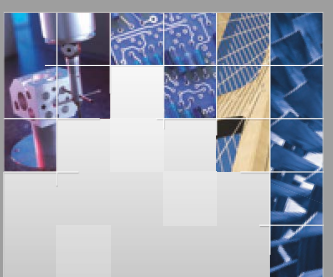

\section{Enfincering}
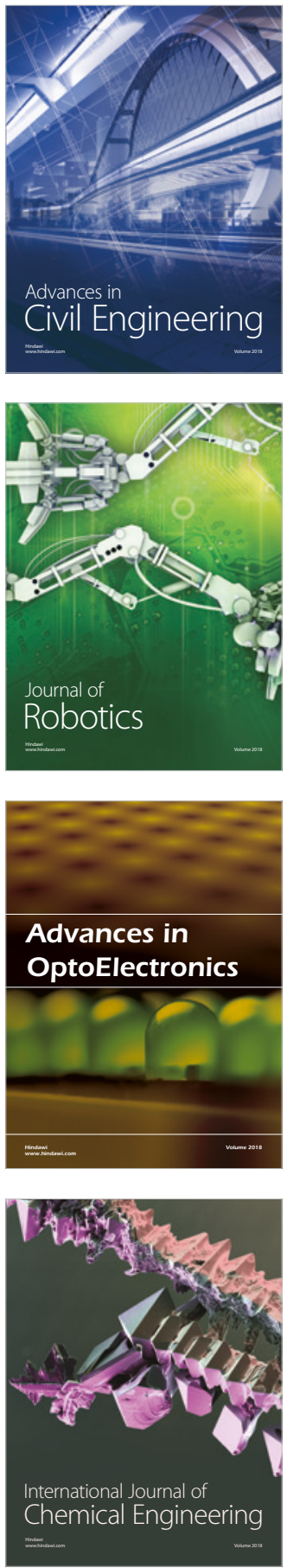

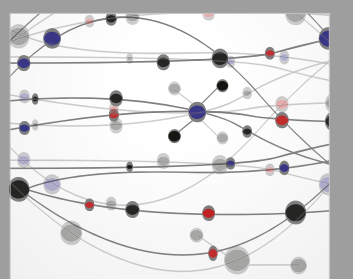

\section{Rotating \\ Machinery}

The Scientific World Journal

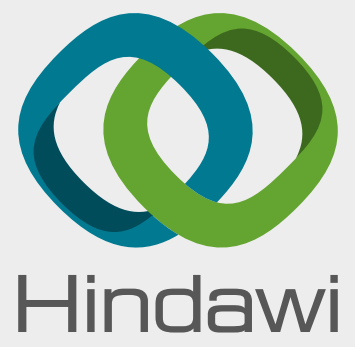

Submit your manuscripts at

www.hindawi.com
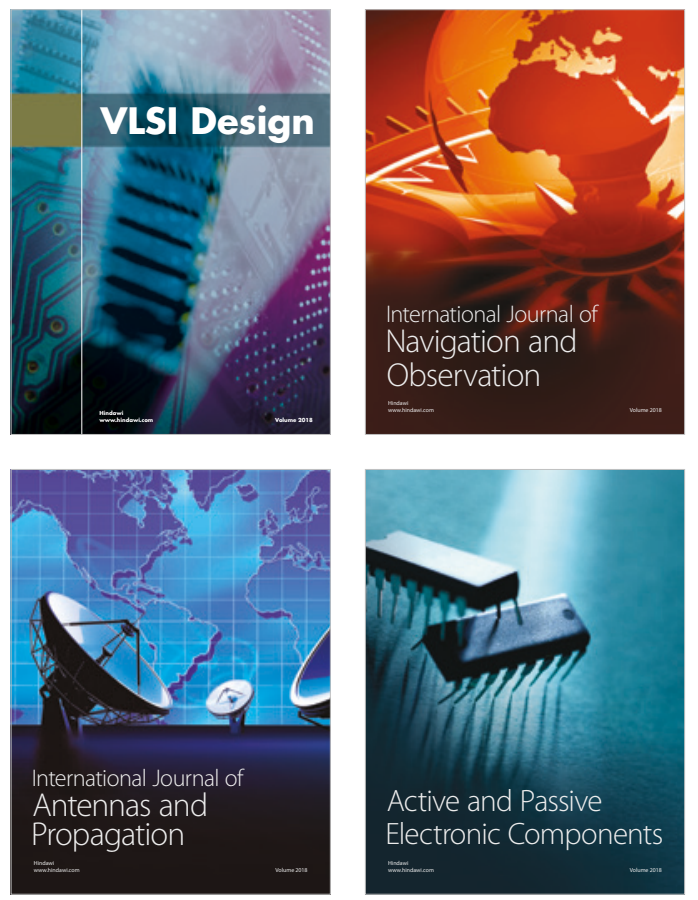
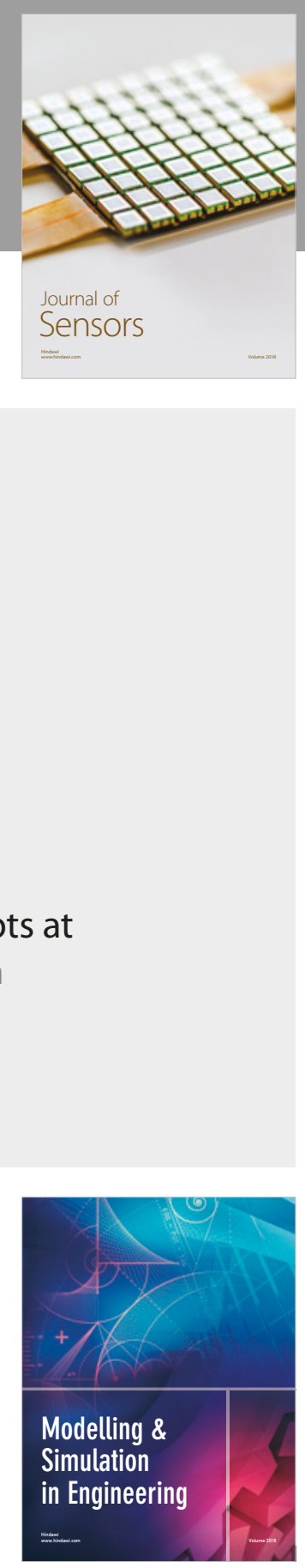

\section{Advances \\ Multimedia}
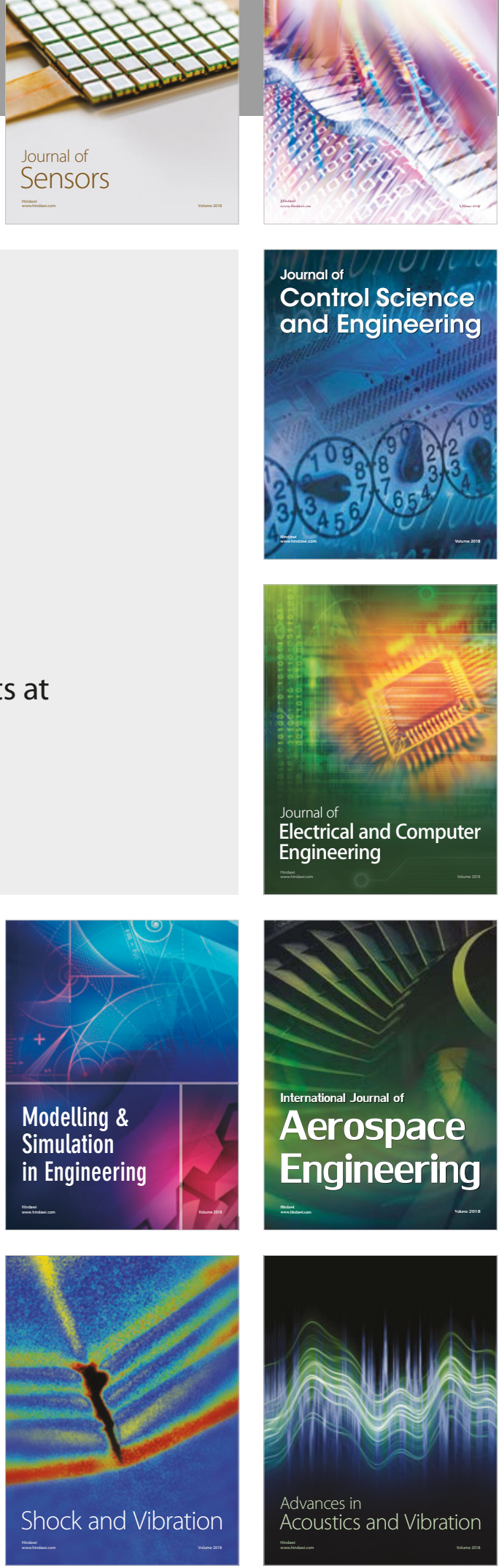